\title{
Study on Association between HbA1c and Lipid profile in Type 2 Diabetic Patients: A Teaching Hospital Based Study
}

\author{
Pallavi Anand', Niranjan Shah ${ }^{2}$, Shrawan ${ }^{3}$, Uday Narayan Singh ${ }^{4}$, P. Satayanarayana ${ }^{5}$ \\ ${ }^{1}$ Associate professor, Department of Biochemistry, Rama Medical College Hospital and Research Centre, Rama City, Mandhana, Kanpur, Uttar Pradesh 209217, \\ ${ }^{2}$ Ph.D. Scholar, Department of Biochemistry, Rama Medical College Hospital and Research Centre, Rama City, Mandhana, Kanpur, Uttar Pradesh 209217, \\ ${ }^{3}$ Professor, Department of Medicine, Rama Medical College Hospital and Research Centre, Rama City, Mandhana, Kanpur, Uttar Pradesh 209217, ${ }^{4}$ Professor \& \\ Head, Department of Biochemistry, Rama Medical College Hospital and Research Centre, Rama City, Mandhana, Kanpur, Uttar Pradesh 209217, ${ }^{5}$ Professor and \\ Dean, Department of Biochemistry, Rama Medical College Hospital and Research Centre, Rama City, Mandhana, Kanpur, Uttar Pradesh 209217.
}

\section{Abstract}

Background: Diabetes mellitus is characterized by chronic hyperglycaemia with disturbances in carbohydrate, fat and protein metabolism resulting from defects in insulin secretion, insulin action or both. Subjects and Methods: type 2 diabetic patients (Age range of 25-70 years) with an age \& sex matched along with 50 healthy controls were selected as per the inclusion and exclusion criteria. Results: The mean age $($ Mean \pm SD) of cases and controls were $48.6 \pm 13.42$ and 30.9 \pm 10.23 years and mean BMI 21.38 $\pm 4.2 \& 25.2 \pm 1.83$ respectively [Table-1]. The Mean \pm SD of cases and controls were FBS (134.62 \pm 20.2$)$, HbA1c (7.8 \pm 2.42$)$, TC (155.6 \pm 31.7$)$, TG (152.3 \pm 26.04$),$ HDL-C (43.4 \pm 8.07$)$ and LDL-C $(81.78 \pm 36.8)$ and FBS $(104 \pm 12.06)$, HbA1c $(5.1 \pm 1.4)$, TC (142.6 \pm 2.5$)$, TG (112.5 \pm 20.3$)$, HDL-C (49.6 \pm 9.5$)$ and LDL-C $(104.06 \pm 17.15)$ respectively[Table-1]. In this study, the level of FBS, HbA1c, TC, TG, LDL-c, were significantly increased $(\mathrm{P}<0.05)$ while HDL-c level was significantly decreased $(\mathrm{P}<0.05)$ as compare to controls. Conclusion: HbA1c showed positive correlation with TC, TG, \& LDL-c and negative correlations were observed between HbA1C and HDL-c.

Keywords: Type-2 DM, FBS, HbA1c and Lipid parameters.

Corresponding Author: Dr. Niranjan Shah, Ph.D. Scholar, Department of Biochemistry, Rama Medical College Hospital and Research Centre, Rama City, Mandhana, Kanpur, Uttar Pradesh -209217.

Received: January 2019

Revised: February 2019

Accepted: March 2019

\section{Introduction}

Diabetes mellitus is characterized by chronic hyperglycaemia with disturbances in carbohydrate, fat and protein metabolism resulting from defects in insulin secretion, insulin action or both. ${ }^{[1]}$ The global figure of people suffering from diabetes mellitus is estimated to rise from current estimate of 415 million to 642 million by 2040. The number of people with type 2 diabetes mellitus is increasing in every country and $75 \%$ of people with diabetes mellitus are living in developing countries. ${ }^{[2]}$ With an increasing incidence worldwide, diabetes mellitus will be a likely leading cause of morbidity and mortality in the future. ${ }^{[3]}$ In Indian population, 61.3 million people had diabetes in 2011, which is expected to reach 101.2 million by 2030 (International Diabetes Federation) now placing India at second position in world diabetic prevalence. ${ }^{[4]}$ The metabolic deregulations associated with diabetes mellitus causes secondary pathophysiological changes in multiple organ systems that impose a tremendous burden on the individual with diabetes and on health care system. Two broad categories of diabetes are designated as type 1 and type 2 diabetes mellitus. Type 2 diabetes mellitus is more common than type $1 \mathrm{DM}$. India is called the diabetic capital of the world. Type 2 diabetes mellitus in Indians is being increasingly seen in younger and less obese persons than in western countries. ${ }^{[5]}$ It is well established that dyslipidemia is a major risk factor for macrovascular complications in patients with type-2 diabetes mellitus and affects 10\%-73\% of this population. ${ }^{[5]}$ Dyslipidemia in diabetes commonly manifests as raised low-density lipoprotein cholesterol (LDL-C), decreased high-density lipoprotein cholesterol (HDL-C) levels, or elevated triglyceride (TG) levels. Furthermore, data from the United Kingdom Prospective Diabetes Study suggest that both decreased HDL-C and elevated LDL-C predict CVD in diabetes. All national and international guidelines recommend aggressive management of lipids in this population. ${ }^{[6,7]}$

Glycated haemoglobin (HbA1c) is a routinely used marker for long-term glycaemic control. Apart from functioning as an indicator for the mean blood glucose level, HbA1c also predicts the risk for the development of diabetic complications in diabetes patients. ${ }^{[8]}$ Many studies have proposed $\mathrm{HbAlc}$ to be used as a biomarker of both 
glycaemic control and dyslipidemia in type 2 diabetes mellitus. ${ }^{[9-11]}$ Thus, the aim of this study was to find any correlation of $\mathrm{HbA} 1 \mathrm{c}$ with lipid profile in patients with type 2 diabetes mellitus.

\section{Subjects and Methods}

This cross sectional study was carried out in the Department of Biochemistry, Rama Medical College Hospital and Research Centre, Kanpur, Uttar Pradesh during the period from January 2017 to December 2018. Randomly selected, 50 type 2 diabetic patients (Age range of 25-70 years) with an age \& sex matched along with 50 healthy controls were selected as per the inclusion and exclusion criteria. Measurement of Biochemical Parameters After an overnight fasting, approximately $5 \mathrm{ml}$ venous blood was collected with the help of disposable syringe. The serum was separated immediately after collecting the blood sample via centrifugation at $2500 \mathrm{rpm}$ for 7 minutes and samples were analysed by following Biochemical parameters: ${ }^{[12]}$

1. Blood Glucose by GOD-POD methods.

2. GlycatedHaemoglobin (HbA1c) by Rapid quantitative test methods.

3. Total Cholesterol (TC) by enzymatic end point CHODPOD methods.

4. Triglyceride (TG) by enzymatic glycerol phosphate oxidase/peroxidase methods.

5. HDL-Cholesterol by direct enzymatic end point method.

6. LDL-Cholesterol by Friedewald's formula. $\{$ LDL-c $=$ Tc-HDL-c(TG/5)\}

Statistical Analysis Standard descriptive statistics (mean \pm standard deviation) were determined for directly measured variable. Unpaired t test were applied to establish the correlations of anthropometric variables, FBG, HbA1c, Lipid profiles, atherogenic indices. The data were evaluated by SPSS statistical package version 16. Pearson's correlation test was performed to examine various correlations. Value of $\mathrm{HbA1c}$ was given as \% of total haemoglobin and values of all other parameters were given in $\mathrm{mg} / \mathrm{dl}$. All values were expressed as mean $\pm \mathrm{SD}$. We used student t-test and pearson's correlation coefficient to find the statistical significance. A P-value $<0.05$ was to be considered statistically significant.

\section{Results \& Discussion}

This present study was conducted in the Department of Biochemistry, Rama Medical College Hospital and Research Centre, Kanpur, Uttar Pradesh. A total of 50 Type 2 Diabetic patients as cases and 50 Healthy subjects' controls were included in this study. The mean age (Mean \pm $\mathrm{SD})$ of cases and controls were $48.6 \pm 13.42$ and $30.9 \pm 10.23$ years and mean BMI 21.38 $\pm 4.2 \& 25.2 \pm 1.83$ respectively [Table-1]. The Mean \pm SD of cases and controls were FBS $(134.62 \pm 20.2), \mathrm{HbA1c}(7.8 \pm 2.42), \mathrm{TC}(155.6 \pm 31.7), \mathrm{TG}$ $(152.3 \pm 26.04)$, HDL-C $(43.4 \pm 8.07)$ and LDL-C $(81.78 \pm$ $36.8)$ and FBS (104 \pm 12.06), HbA1c $(5.1 \pm 1.4)$, TC
(142.6 \pm 2.5$)$, TG (112.5 \pm 20.3$)$, HDL-C (49.6 \pm 9.5) and LDL-C (104.06 \pm 17.15$)$ respectively[Table-1]. In this study, the level of FBS, HbA1c, TC, TG, LDL-c, were significantly increased $(\mathrm{P}<0.05)$ while HDL-c level was significantly decreased $(\mathrm{P}<0.05)$ as compare to controls.

Table 1: Comparison of Biochemical parameters between cases and Controls

\begin{tabular}{|c|c|c|}
\hline \multirow[t]{2}{*}{$\begin{array}{l}\text { Biochemical } \\
\text { Parameters }\end{array}$} & $\begin{array}{l}\text { Type } 2 \text { DM } \\
(50)\end{array}$ & $\begin{array}{l}\text { Healthy Controls } \\
(50)\end{array}$ \\
\hline & Mean \pm SD & Mean \pm SD \\
\hline Age in years & $48.6 \pm 13.42$ & $30.9 \pm 10.23$ \\
\hline BMI & $21.38 \pm 4.2$ & $25.2 \pm 1.83$ \\
\hline FBS (mg/dl) & $134.62 \pm 20.2$ & $104 \pm 12.06$ \\
\hline HbA1c (\%) & $7.8 \pm 2.42$ & $5.1 \pm 1.4$ \\
\hline $\mathrm{TC}(\mathrm{mg} / \mathrm{dl})$ & $155.6 \pm 31.7$ & $142.6 \pm 2.5$ \\
\hline $\mathrm{TG}(\mathrm{mg} / \mathrm{dl})$ & $152.3 \pm 26.04$ & $112.5 \pm 20.3$ \\
\hline HDL-c(mg/dl) & $43.4 \pm 8.07$ & $49.6 \pm 9.5$ \\
\hline LDL-c(mg/dl) & $81.78 \pm 36.8$ & $104.06 \pm 17.15$ \\
\hline
\end{tabular}

Statistically Significant at $\mathrm{p}$ value $<0.05$

[Table 2] shows the results of association between TC, TG, HDL-c, LDL-c, and with HbA1c levels of patients and control group. There was a significant positive correlation between HbA1c and TC $(r=+0.46, p=0.000)$. HbA1c also demonstrated a significant correlation with $\mathrm{TG}(\mathrm{r}=+0.34$, $\mathrm{p}$ $=0.01$ ). The correlation between HbA1c and HDL-C was negative and was found to be statistically not significant ( $\mathrm{r}$ $=-0.015, \mathrm{p}=0.91)$. Furthermore, it was found that HbA1c was positively and significantly related to LDL-C $(\mathrm{r}=$ $+0.41, \mathrm{p}=0.003)$.

Table 2: Shows the Correlation between HbA1c and Lipid parameters type 2 diabetic patients.

\begin{tabular}{|l|c|c|}
\hline Parameters & Correlation Coefficient(r) & P- value \\
\hline TC & +0.46 & 0.000 \\
\hline TG & +0.34 & 0.01 \\
\hline HDL-c & -0.015 & $0.91^{*}$ \\
\hline LDL-c & +0.41 & 0.003 \\
\hline
\end{tabular}

Statistically Significant at p value $<0.05 ; *$ NS: Statistically not Significant

High prevalence of hypercholesterolemia, hypertriglyceridemia and high LDL-c \& low HDL-c was found in type- 2 diabetes which is well known risk factors for cardiovascular disease. ${ }^{[13]}$ The cause of dyslipidemia in type 2 diabetes mellitus may be that, insulin is not working properly and which affects the liver apo lipoprotein production. ${ }^{[14]}$ The apo lipoprotein regulates the enzymatic activity of lipoprotein lipase and cholesterol ester transport protein. ${ }^{[14]}$ A highly positive significant relationship of HbA1c with dyslipidemia was observed in the present study. Erciyas et al, ${ }^{[15]}$ also reported positive correlation of HbA1c level with TC and TG in diabetic patients. The diabetes complication and control trail established HbA1c as Value $\leq 7.0 \%$ was said to be appropriate for reducing the risk of cardiovascular complications. The diabetic patients with higher $\mathrm{HbA1c}$ value can exhibit a significant increase in TC, TG, LDL-c and HDL-c in comparison to patients with $\mathrm{HbA} 1 \mathrm{c}$ value $\leq 7.0 \%$ khan et ${ }^{\left[1,{ }^{[16,17]}\right.}$ reported the severity of dyslipidemia increases in patients with higher $\mathrm{HbA1c}$ value. As elevated HbA1c and dyslipidemia are independent risk factors of CVD, diabetic patients with 
elevated HbA1c and dyslipidemia can be considered as a very high risk group for CVD. Improving glycemic control can substantially reduce the risk of cardiovascular events in diabetes and also reported that reducing the HbA1c level by $0.2 \%$ could lower the mortality by $10 \% .^{[18,19]}$ Insulin plays an important role in production of liver apo-lipoprotein which further modulates enzymatic activity of lipoprotein lipase and cholesterol ester transport protein. This mechanism could be responsible for dyslipidaemia in Diabetes. ${ }^{[20]}$ Also, insulin deficiency reduces the activity of hepatic lipase and therefore several steps in the production of biologically active lipoprotein lipase may also be altered in Diabetes. ${ }^{[21]}$ Thus present study suggests the importance of glycemic control in prevention of cardiovascular disease in type-2 diabetics.

\section{Conclusion}

In conclusion, the HbA1c showed positive correlation with TC, TG, \& LDL-c and negative correlations were observed between HbA1C and HDL-c. So HbA1c can be used as an indirect indicator of dyslipidaemia in type 2 diabetes in addition to as glycemic control biomarker. Thus early diagnosis of dyslipidaemia can be used as a preventive measure for development of cardiovascular disease in patients with type 2 diabetes mellitus. So, HbA1c may be utilized for screening diabetic patient for risk of cardiovascular events and also for timely intervention with lipid lowering drugs.

\section{References}

1. Bennett HP, Knowler WC, Definition, Classification of Diabetes Mellitus and Glucose Homeostasis. In CR Kahn, GC Weir, GL King, AC Moses, RJ Smith and AM Jacobson editors. Joslin's Diabetes Mellitus, Philadelphia: LWW; 200,331.

2. Powers AC, Diabetes Mellitus: Complications. In DL Kasper, AS Fauci, DL Longo, SL Hauser, JL Jameson and J Loscalzo editors. Harrison's Principles of Internal Medicine. New York: McGraw-Hill Education; 2015. p.2399.

3. Diabetes: facts and figures [Internet]. International Diabetes Federation. [cited 2016Jul14]. Available from: http://www. idf.org/about-diabetes/facts-figures.

4. Lt Gen SR Mehta, VSM, Col AS Kashyap, Lt Col S Das. Diabetes Mellitus in India: The Modern Scourge. MJAFI 2009;65:50-4.

5. Mukhopadhyay J, Kanjilal S, Biswas M. Diabetic dyslipidemia- priorities and targets in India. [Internet]. http://www.apiindia.org/content_mu_2010.html. 2013 [cited 23 August 2016].

6. U.K. Prospective Diabetes Study 32. Ethnicity and cardiovascular disease. The incidence of myocardial infarction in white, South Asian, and Afro-Caribbean patients with type 2 diabetes Diabetes Care. 1998; 21:1271- 7 .

7. Sarat Chandra K, Bansal M, Nair T, et al. Consensus statement on management of dyslipidemia in Indian subjects. Indian Heart Journal. 2014;66(Suppl 3):S1-S51.

8. Selvin E. Meta-Analysis: Glycosylated hemoglobin and cardiovascular disease in diabetes mellitus. Annals of Internal Medicine. 2004; $141: 421$.

9. Lodha R, Lal R, Biyani $\mathrm{S}$. HbA1c as screening biomarker of dyslipidemia in type 2 diabetes mellitus patients. Scholar journal of applied medicsl sciences. 2016;4:1600-1602.

10. Alan R, Verma P. Glycated hemoglobin as a dual biomarker in type 2 diabetes mellitus predicting glycemic control and dyslipidemia risk. International journal of life science scientific research. 2015;1:62-65.

11. Parveen A, Chimkode SM, Kumaran SD. Correlation of HbA1c levels with serum lipid profile in patients with type 2 diabetes mellitus. Research journal of pharmaceutical, biological and chemical sciences. 2015;6:703-706.

12. Manoj Kr Yadav, Tapan Kr Mohapatra, Rabindra Kr Mohapatraet. al. Study on Glycated Hemoglobin \& lipid profile in Type-2 Diabetes Mellitus. International Journal of Science \& Research (IJSR) 2015;4(6): 1917-19.

13. American Diabetes association. Dyslipidemia management in adult with diabetes. Diabetes care 2004; 24: s68-s71.

14. Goldberg IJ. Lipoprotein lipase and lipolysis: central roles in lipoprotein metabolism and atherogenesis. J. lipid Res 1996; 37: $693-$ 707. Pmid: 8732771.

15. Erciyas F, taneli F, Arslan B and Uslu Y. glycemic control, oxidative stress and lipid profile in children with type I diabetes mellitus, Arch. Med. Res 2004; 35: 134- 140.

16. Rohlfing CL, Wiedmeyer HM, Little RR, England JD, Tennill A and Goldstein DE. Definging the relationship between plasma glucose and HbA1c: analysis of glucose profiles and HbA1c in the Diabetes Control and Complications trail. Diabetes care 2002;25:275-278.

17. Khan HA, Sobki SH and Khan SA. Association between glycemic control and serum lipids profile in type 2 diabetic patients: HbA1c predicts dyslipidemia. Clin. Exp. Med 2007;7:24- 29.

18. Rosediani M, Azidah AK, Mafauzy M. correlation between fasting plasma glucose, post prandial glucose and gycated hemoglobin and fructosamine. Med J Malaysia 2006;61:67-71.pmid:16708736.

19. Smith SC. Multiple risk factors for cardiovascular disease and diabetes mellitus. Am J med 2007;120:s3- s11.

20. Goldberg IJ. Lipoprotein lipase and lipolysis: central roles in lipoprotein metabolism and atherogenesis. J Lipid Res1996; 37: 693707.

21. Tavangar K, Murata Y, Pedersen ME, Goers JF, Hoff-man AR, Kraemer FB. Regulation of lipoprotein lipase in thediabetic rat. J Clin Invest 1992; 90: 1672-1678.

Copyright: () the author(s), 2019. It is an open-access article distributed under the terms of the Creative Commons Attribution License (CC BY 4.0), which permits authors to retain ownership of the copyright for their content, and allow anyone to download, reuse, reprint, modify, distribute and/or copy the content as long as the original authors and source are cited.

How to cite this article: Anand P, Shah N, Shrawan, Singh UN, Satayanarayana P. Study on Association between HbA1c and Lipid profile in Type 2 Diabetic Patients: A Teaching Hospital Based Study. Asian J. Med. Res. 2019;8(1):BC17-BC19.

DOI: dx.doi.org/10.21276/ajmr.2019.8.1.BC17

Source of Support: Nil, Conflict of Interest: None declared. 